Stoeber, J., Haskew, A. E., \& Scott, C. (2015). Perfectionism and exam performance: The mediating effect of task-approach goals. Personality and Individual Differences, 74, 171-176. DOI: $10.1016 /$ j.paid.2014.10.016

Perfectionism and Exam Performance:

The Mediating Effect of Task-Approach Goals

\author{
Joachim Stoeber \\ Amy E. Haskew \\ Charlotte Scott \\ University of Kent
}

\begin{abstract}
Author Note
Joachim Stoeber, Amy Haskew, and Charlotte Scott, School of Psychology, University of
\end{abstract}

Kent.

Correspondence concerning this article should be addressed to Joachim Stoeber, School of Psychology, University of Kent, Canterbury, Kent CT2 7NP, United Kingdom. Phone: +44-1227824196; fax: +44-1227-827030; e-mail: J.Stoeber@kent.ac. 


\begin{abstract}
Perfectionistic strivings are positively correlated with students' achievement goals and exam performance. However, so far no study has employed a prospective design investigating whether achievement goals mediate the positive relationship between perfectionistic strivings and exam performance. In the present study, 100 university students completed a measure of self-oriented perfectionism and socially prescribed perfectionism (Hewitt \& Flett, 1991) and received a chapter from a textbook to study for 2-4 days. Then they returned to the lab to complete a measure of achievement goals following the $3 \times 2$ model (Elliot, Murayama, \& Pekrun, 2011) and sit a mock exam testing their knowledge of the chapter. Multiple regressions showed that socially prescribed perfectionism negatively predicted exam performance when the overlap with self-oriented perfectionism was controlled for. In contrast, self-oriented perfectionism - a defining indicator of perfectionistic strivings - positively predicted exam performance. Moreover, task-approach goals mediated the positive relationship between self-oriented perfectionism and exam performance. The findings suggest that perfectionistic strivings make students adopt task-approach goals that help them achieve better results on exams.
\end{abstract}

Keywords: perfectionism; $3 \times 2$ achievement goals; academic achievement; exam performance; multiple choice tests; mediation analysis; suppression

\title{
1.1 Perfectionism
}

\section{Introduction}

Perfectionism is a personality disposition characterized by striving for flawlessness and setting exceedingly high standards of performance accompanied by overly critical evaluations of one's behavior (Frost, Marten, Lahart, \& Rosenblate, 1990; Hewitt \& Flett, 1991). However, perfectionism has various aspects, and there are different dimensions of perfectionism with different characteristics. Consequently, perfectionism is best conceptualized as a multidimensional personality disposition (Enns \& Cox, 2002).

Regarding multidimensional conceptions of perfectionism, one of the most influential and widely researched models is Hewitt and Flett's (1991) model of perfectionism. Acknowledging that perfectionism has personal and social aspects, the model differentiates two main dimensions of perfectionism: self-oriented perfectionism and socially prescribed perfectionism. ${ }^{1}$ Selforiented perfectionism encompasses internally motivated beliefs that striving for perfection and being perfect are important. Self-oriented perfectionists have exceedingly high personal standards, strive for perfection, and expect to be perfect. In contrast, socially prescribed perfectionism encompasses externally motivated beliefs that striving for perfection and being perfect are important to others. Socially prescribed perfectionists believe that others expect them to be perfect and that they have to meet these expectations (Hewitt \& Flett, 1991, 2004).

Factor analyses comparing various measures of multidimensional perfectionism found two superordinate factors underlying the different dimensions of perfectionism: perfectionistic strivings and perfectionistic concerns (Stoeber \& Otto, 2006). In these analyses, self-oriented perfectionism always emerged as a defining indicator of perfectionistic strivings whereas socially prescribed perfectionism emerged as a defining indicator of perfectionistic concerns. Further research established that perfectionistic concerns consistently showed positive correlations with negative characteristics, processes, and outcomes (e.g., neuroticism, maladaptive coping, negative affect) indicating that perfectionistic concerns capture maladaptive aspects of perfectionism. In contrast, perfectionistic strivings often showed positive correlations with positive characteristics, 
processes, and outcomes (e.g., conscientiousness, adaptive coping, positive affect) - particularly when the overlap with perfectionistic concerns was controlled for (Hill, Huelsman, \& Araujo, 2010) — suggesting that perfectionistic strivings capture adaptive aspects of perfectionism (see Stoeber \& Otto, 2006, for a review).

\subsection{Perfectionism, Academic Achievement, and Exam Performance}

The differentiation between perfectionistic strivings and perfectionistic concerns is also critical when regarding the relationships of perfectionism and academic achievement. The reason is that perfectionistic strivings show positive relationships with indicators of academic achievement such as grade point average and exam performance (see Stoeber, 2012, for a review). In contrast, the relationships between perfectionistic concerns and academic performance are less clear. Most studies failed to find negative relationships between perfectionistic concerns and academic performance, except when discrepancy - that is, perfectionists' perceptions that they are failing to meet their high standards (Slaney, Rice, Mobley, Trippi, \& Ashby, 2001)—was used as an indicator of perfectionistic concerns. Furthermore, Flett, Blankstein, and Hewitt (2009) found socially prescribed perfectionism to negatively predict students' performance in a classroom exam involving a multiple choice test. In contrast, self-oriented perfectionism showed the expected positive effect on exam performance.

\subsection{Limitations of Previous Studies}

There are, however, some open questions. First, the vast majority of studies investigating perfectionism and academic performance used cross-sectional correlational designs (Stoeber, 2012). This leaves open the question of the direction of the relationships because it is conceivable that higher academic achievement may not be an effect, but a precursor of perfectionistic strivings: Students who receive top marks may develop perfectionistic personal standards and expectations as a consequence of high academic achievement (Flett, Hewitt, Oliver, \& Macdonald, 2002).

Second, no study so far has investigated what processes may be responsible for the perfectionism-achievement relationships. One possibility is that students' achievement goals are responsible, following findings from research on perfectionism and sport performance. Using a prospective correlational design, Stoeber, Uphill, and Hotham (2009) measured perfectionistic strivings and perfectionistic concerns one day before athletes competed in a race. In addition, they measured athletes' achievement goals for the race following the $2 \times 2$ model of achievement goals (Elliot \& McGregor, 2001). The model has two dimensions: definition and valence. Definition captures the content of achievement goals differentiating performance and mastery. Valence captures the orientation of achievement goals differentiating approach and avoidance. Hence the $2 \times 2$ model distinguishes four goals: performance-approach (striving to do better than others), performance-avoidance (avoiding doing worse than others), mastery-approach (striving to master the task or to do better than one has done before), and mastery-avoidance goals (avoiding not being able to master the task or doing worse than one has done before). Stoeber and colleagues (2009) found that perfectionistic strivings predicted better race results. Moreover, the effect of perfectionistic strivings was mediated by athletes' achievement goals: Athletes high in perfectionistic strivings showed higher levels of performance-approach goals relative to performance-avoidance goals when compared to athletes low in perfectionistic strivings, and the difference between performance-approach and -avoidance goals mediated the positive effect of perfectionistic strivings on race performance. 


\subsection{The Present Study}

Against this background, the aim of the present study was to provide a first investigation of how multidimensional perfectionism and achievement goals predict exam performance using a prospective design. In this, self-oriented perfectionism and socially prescribed perfectionism served as indicators of perfectionistic strivings and perfectionistic concerns (Stoeber \& Otto, 2006), achievement goals were measured following the $3 \times 2$ model (Elliot, Murayama, \& Pekrun, 2011), and exam performance was measured by having students sit a mock exam comprised of a multiple choice test (cf. Flett et al., 2009).

The reason why we followed the $3 \times 2$ model (instead of the $2 \times 2$ model used in previous research) was that the $3 \times 2$ model was developed in response to criticism that the $2 \times 2$ model did not differentiate between absolute and intrapersonal standards of comparison (e.g., striving to master the task versus striving to do better than one has done before). Consequently, the $3 \times 2$ model introduced a tripartite differentiation to the definition dimension capturing absolute (task), intrapersonal (self), and interpersonal (others) standards of comparison. Hence the model distinguishes six goals: task-approach (striving to master the task), task-avoidance (avoiding not being able to master the task), self-approach (striving to do better than one has done before), selfavoidance (avoiding to do worse than one has done before), other-approach (striving to do better than others), and other-avoidance goals (avoiding to do worse than others). Examining how the goals predicted students' classroom behavior and academic performance, Elliot and colleagues (2011) found preliminary evidence supporting the $3 \times 2$ model. Task-approach goals positively predicted intrinsic motivation and learning efficacy whereas self-approach goals predicted energy in class and other-approach goals predicted exam performance. In contrast, self-avoidance goals negatively predicted energy in class whereas other-avoidance goals negatively predicted learning efficacy and exam performance and positively predicted worry about exams. (Task-avoidance goals made no unique predictions.)

Based on previous research on perfectionism and academic performance (Stoeber, 2012), we expected self-oriented perfectionism to positively predict exam performance and socially prescribed perfectionism to either show no relationship with exam performance or negatively predict exam performance (cf. Flett et al., 2009). Regarding the $3 \times 2$ achievement goals, we expected other-approach goals to positively predict exam performance (cf. Elliot et al., 2011) in line with previous research on the $2 \times 2$ model suggesting that performance-approach goals predict academic achievement (Moller \& Elliot, 2006). However, because our design included a learning component (participants had to learn a new text for the mock exam; see 2.3.2.), there was also the possibility that task-approach goals, which have been associated with learning efficacy (Elliot et al., 2011; Siu-Man \& Leung, 2014), would positively predict exam performance.

\subsection{Participants}

\section{Method}

A sample of 100 undergraduate psychology students (11 male, 89 female) was recruited via the School of Psychology's research participation scheme (RPS). Mean age of students was 19.9 years $(S D=2.7)$. Students volunteered to participate in the study for extra course credit.

2.2 Design and procedure

The study followed a prospective correlational design with two measurement points: Time 1 (T1) and Time 2 (T2). All measures were completed online using the School's secure Qualtrics ${ }^{\circledR}$ platform, except the mock exam which-to simulate an actual exam-was presented 
as a paper-and-pencil multiple choice test. At T1, participants followed the link from the RPS webpage to the Qualtrics ${ }^{\circledR}$ platform to complete the perfectionism measure. Afterwards they were sent an email with a PDF copy of the text to study for the mock exam. In addition, the email requested that they make an appointment with the experimenter (the second or third author) to come to the lab 2 or 3 days later (T2) to complete another questionnaire and take the exam. In the lab, participants first completed the achievement goals measure (T2.1), which was again presented online, and then sat the mock exam, which was presented as a paper-and-pencil test (T2.2).

On average, participants came to the lab 2.3 days $(S D=0.5$; range $=1.7-4.1$ days $)$ after they received the text to study. Because lab space was only available at certain days and times (excluding weekends and evenings), a few participants had less than 2 or more than 3 days between receiving the text and their lab appointment. The number of days between T1 and T2, however, showed no significant correlations with any of the study's variables, so it was not further regarded.

\subsection{Materials}

\subsubsection{Perfectionism}

To measure perfectionism, we used the 30 items of the Multidimensional Perfectionism Scale (MPS; Hewitt \& Flett, 1991) capturing self-oriented perfectionism (15 items; e.g., "I demand nothing less than perfection of myself') and socially prescribed perfectionism (15 items; e.g., "People expect nothing less than perfection from me"). The MPS is a widely used measure of multidimensional perfectionism and has shown reliability and validity in numerous studies (Hewitt \& Flett, 2004). The items were presented using the MPS's standard instruction ("Listed below are a number of statements concerning personal characteristics and traits...”), and participants responded on a scale from 1 (strongly disagree) to 7 (strongly agree).

\subsubsection{Text to study}

As a text to study, participants received 10 pages of a chapter on forgiveness from a textbook on personality and individual differences aimed at undergraduate students ("Introducing forgiveness"; Maltby, Day, \& Macaskill, 2010, pp. 545-554). The chapter was chosen because forgiveness was not taught on any undergraduate courses in psychology, so participants had no prior knowledge of the chapter's content. Participants were instructed to study the text in the same way as they would in preparation for an actual exam. Moreover, they were informed that the exam would contain 20 multiple choice questions following the same format used in actual exams.

\subsubsection{Achievement goals}

To measure achievement goals, we used the $3 \times 2$ Achievement Goal Questionnaire (AGQ; Elliot et al., 2011) which comprises 18 items capturing task-approach goals (3 items; e.g., "To get a lot of questions right on the exams in this class"), task-avoidance goals (3 items; e.g., "To avoid incorrect answers on the exams in this class"), self-approach goals (3 items; e.g., "To perform better on the exams in this class than I have done in the past on these types of exams"), selfavoidance goals ( 3 items; e.g., "To avoid doing worse on the exams in this class than I normally do on these types of exams"), other-approach goals (3 items; e.g., "To outperform other students on the exams in this class"), and other-avoidance goals (3 items; e.g., "To avoid doing worse than other students on the exams in this class"). To our knowledge, the AGQ has so far been used in only three published studies all of which attest to the scales' reliability and validity (Elliot et al., 
2011; Siu-Man \& Leung, 2014; Yang \& Cao, 2013). To adapt the items for the present study, we changed "exams" (plural) to "exam" (singular) and removed "in this class" from all items (e.g., "To get a lot of questions right on the exams in this class" became "To get a lot of questions right on the exam"). The instructions were changed accordingly (i.e., "The following statements represent types of goals that you may or may not have for this exam..."), and participants responded on a scale from 1 (not true of me) to 7 (extremely true of me).

\subsubsection{Exam performance}

To measure exam performance, a 20-item multiple choice test (MCT) was constructed to test participants' knowledge of the text book chapter they were instructed to study. For this, we used the same format that the School of Psychology uses in actual exams, namely four choices per question of which only one is correct (e.g., "In Worthington's REACH model of forgiveness, what does the ' $R$ ' in the acronym stand for?" with the four choices "a. Recognize need for forgiveness," "b. Recover from hurt," "c. Recall the hurt," and "d. Relate to hurtful behavior"; correct choice $=\mathrm{c}){ }^{2}$ The MCT was piloted prior to the study to ensure all questions were comprehensible and neither too easy nor too difficult and to estimate the time participants would need. As a result of these pilots, participants were given 30 minutes to complete the MCT. On average, participants took 12.0 minutes $(S D=3.9$; range $=4-30)$ and answered 13.0 questions correctly $(S D=3.6$; range $=5-20)$.

\subsection{Preliminary analyses}

First, we computed scale scores by averaging responses across items except for the exam where we computed sum scores (number of correct answers). Next, we examined whether the variance-covariance matrices of males and females differed by computing a Box's $M$ test with gender as between-participants factor. Because Box's $M$ is highly sensitive to even minor differences, it is tested against $p<.001$ (Tabachnick \& Fidell, 2007). The test was nonsignificant (Box's $M=91.94, F[45,1009]=1.30, p=.089$ ). Consequently, analyses were collapsed across gender. Finally, we examined the scores' reliability. All scores showed Cronbach's alphas $>.70$ indicating satisfactory reliability (see Table 1).

\subsection{Correlations}

\section{Results}

First, we examined the bivariate correlations between perfectionism at T1, achievement goals at T2.1, and exam performance at T2.2 (see Table 1). As expected, self-oriented perfectionism showed positive correlations with all approach goals (task-, self-, other-approach). Furthermore, it showed a positive correlation with exam performance as did other-approach goals. In addition, task-approach goals showed a positive correlation with exam performance.

Unexpectedly, self-oriented perfectionism also showed positive correlations with all avoidance goals (task-, self-, other-avoidance) whereas socially prescribed perfectionism showed a positive correlation with other-approach goals, but not with any avoidance goals. Moreover, all avoidance goals showed positive correlations with exam performance.

However, note that-like the two dimensions of perfectionism-the achievement goals showed significant overlap as indicated by their large-sized positive intercorrelations. Therefore we decided to conduct multiple regression analyses to examine which of the relationships with exam performance that the bivariate correlations suggested were unique and which variables would continue to be significant predictors once the overlap between the achievement goals was controlled for. 


\subsection{Regression analyses}

In the first analysis (Regression Analysis 1), exam performance was the criterion, and all achievement goals were entered simultaneously as predictors. In the second analysis (Regression Analysis 2), exam performance was again the criterion, but the analyses comprised two separate steps. In Step 1, the two perfectionism dimensions were entered simultaneously to examine how perfectionism predicted exam performance. In Step 2, the achievement goals were entered simultaneously to examine which achievement goals predicted unique variance in exam performance above perfectionism. Because the predictors showed substantial intercorrelations, we controlled for multicollinearity by checking if any predictor's variance inflation factor (VIF) exceeded the critical value of 10 (Kutner, Nachtsheim, \& Neter, 2004). All predictors showed VIFs $<4.07$ indicating that multicollinearity was not an issue.

Table 2 shows the results of the regression analyses. In Regression Analysis 1, only taskapproach goals predicted exam performance with a positive regression weight once the overlap between the achievement goals was controlled for, indicating that task-approach goals were a unique predictor of exam performance. In Step 1 of Regression Analysis 2, self-oriented perfectionism showed a positive regression weight in the prediction of exam performance. In addition, socially prescribed perfectionism showed a negative regression weight. In Step 2, only task-approach goals showed a significant regression weight positively predicting exam performance above perfectionism. Furthermore, self-oriented perfectionism ceased to show a significant regression weight suggesting that task-approach goals mediated the positive relationship between self-oriented perfectionism and exam performance (Baron \& Kenny, 1986).

\subsection{Mediation analysis}

To test whether this was the case, we conducted a mediation analysis following the procedures of Preacher and Hayes (2004) with self-oriented perfectionism as the independent variable, task-approach goals as the mediator, and exam performance as the dependent variable. The Sobel test of the mediation effect was significant with $z=2.40, p<.05$, and the $95 \%$ confidence interval $[\mathrm{CI}]$ from the bootstrap test of the indirect effect (5,000 bootstraps) did not include zero indicating that the indirect effect was significant. Task-approach goals mediated the relationship between self-oriented perfectionism and exam performance (see Figure 1).

Because Campbell and DiPaula (2002) found self-oriented perfectionism to comprise two aspects showing differential validities - perfectionistic striving and importance of being perfect (see Stoeber \& Childs, 2010, for details) - we conducted additional analyses to examine if the effect of self-oriented perfectionism was an effect of perfectionistic striving or importance of being perfect. Results showed that importance of being perfect did not predict exam performance $(\beta=.12, n s)$. In contrast, perfectionistic striving showed the exact same pattern as self-oriented perfectionism. Perfectionistic striving predicted task-approach goals $(\beta=.33, p<.001)$ and exam performance $(\beta=.27, p<.01)$ with the latter path reduced to $\beta=.16, n s$ when task-approach goals were controlled for (Sobel $z=2.39, p<.05$; bootstrap 95\% CI not including zero). Hence the effect shown in Figure 1 appeared to be an effect of the perfectionistic striving aspect of selforiented perfectionism.

\section{Discussion}

The aim of this study was to provide a first investigation of how multidimensional perfectionism and achievement goals predict exam performance using a prospective design and examining achievement goals following the $3 \times 2$ model (Elliot et al., 2011). In line with previous findings that perfectionistic strivings show positive relationships with academic achievement, we 
found that self-oriented perfectionism positively predicted exam performance. Students who showed higher levels of self-oriented perfectionism before receiving a text to study for a mock exam achieved better results 2-3 days later when sitting the exam when compared to students who showed lower levels of self-oriented perfectionism. Furthermore, we found that taskapproach goals mediated the relationship between self-oriented perfectionism and exam performance. Students high in self-oriented perfectionism showed higher levels of task-approach goals before sitting the exam which explained the positive relationship between self-oriented perfectionism and exam performance.

In addition, we found that socially prescribed perfectionism was unrelated to exam performance when bivariate correlations were regarded, but negatively predicted exam performance when the overlap with self-oriented perfectionism was controlled for. This finding is noteworthy because we know of only one published study that found a negative relationship between socially prescribed perfectionism and exam performance (Flett et al., 2009). Moreover, the finding indicates that the overlap between perfectionistic strivings and perfectionistic concerns not only suppresses perfectionistic strivings' positive associations (Stoeber \& Otto, 2006), but may also suppress perfectionistic concerns' negative associations (Hill et al., 2010). Only when the positive influence of self-oriented perfectionism was controlled for did socially prescribed perfectionism show a negative association with exam performance.

Moreover, there were two unexpected findings we found noteworthy. First, self-oriented perfectionism showed positive correlations not only with all approach goals, but also with all avoidance goals, even when partial correlations were computed controlling for the overlap with socially prescribed perfectionism. ${ }^{3}$ Whereas this finding is not in line with previous research that found perfectionistic strivings in athletes to positively predict approach goals, but not avoidance goals once the overlap with perfectionistic concerns was controlled for (e.g., Stoeber, Stoll, Salmi, \& Tiikkaja, 2009), it dovetails with a recent study that found self-oriented perfectionism in school students to show positive relationships with performance- and mastery-avoidance goals even after controlling for the overlap with socially prescribed perfectionism (Damian, Stoeber, Negru, \& Băban, 2014). Together with the present findings, this suggest that self-oriented perfectionism makes students adopt both approach and avoidance goals in their pursuit of academic achievement. Second, differently from Elliot et al. (2011), we found that task-approach goals positively predicted exam performance, not other-approach goals. One possible explanation for this discrepant finding is that, in our study, students were required to study the content of a textbook chapter they were unfamiliar with and then sit a mock exam testing their knowledge of the chapter's content. Consequently, the study had a strong learning component favoring students who endorsed task-approach goals which have been shown to predict learning efficacy (Elliot et al., 2011; Siu-Man \& Leung, 2014). This explanation, however, is speculative, and future studies will need to replicate our finding to confirm its validity.

The present study had further limitations. First, the sample was predominantly female. Whereas the gender distribution was representative for students in psychology, future studies may aim at sampling students in subjects that have a greater gender balance (e.g., biological sciences). Second, the study investigated performance in a mock exam, not an actual exam. Whereas the multiple choice test of the mock exam had the same format as an actual exam, it is unclear how well participants prepared for the exam and how seriously they took the test. Consequently, future studies need to reproduce the present findings with actual exams (cf. Elliot et al., 2011; Flett et al., 2009). In addition, future studies examining the relationships of perfectionism and exam performance should include conscientiousness as a control variable (cf. Noftle \& Robins, 2007). Finally, it is unclear why socially prescribed perfectionism did not show any significant 
correlations with the avoidance goals of the $3 \times 2$ model considering that previous research found socially prescribed perfectionism in students to show unique positive relationships with avoidance goals, particularly performance-avoidance goals (Damian et al., 2014). Consequently, further research is required including other measures of perfectionistic concerns (cf. Stoeber \& Otto, 2006) to reinvestigate the relationships of perfectionistic concerns and the $3 \times 2$ achievement goals in students.

Despite these limitations, the findings of the present study make an important contribution to the literature on perfectionism and academic achievement because they expand on previous findings on perfectionism, achievement goals, and exam performance indicating that perfectionistic strivings predict better exam performance and that the endorsement of taskapproach goals may explain why perfectionistic strivings lead to higher performance.

\section{References}

Baron, R. M., \& Kenny, D. A. (1986). The moderator-mediator variable distinction in social psychological research: Conceptual, strategic, and statistical considerations. Journal of Personality and Social Psychology, 51, 1173-1182.

Campbell, J. D., \& Di Paula, A. (2002). Perfectionistic self-beliefs: Their relation to personality and goal pursuit. In G. L. Flett \& P. L. Hewitt (Eds.), Perfectionism (pp. 181-198). Washington, DC: APA.

Damian, L. E., Stoeber, J., Negru, O., \& Băban, A. (2014). Perfectionism and achievement goal orientations in adolescent school students. Psychology in the Schools, 51, 960-971.

Enns, M. W., \& Cox, B. J. (2002). The nature and assessment of perfectionism: A critical analysis. In G. L. Flett \& P. L. Hewitt (Eds.), Perfectionism (pp. 33-62). Washington, DC: APA.

Elliot, A. J., \& McGregor, H. A. (2001). A $2 \times 2$ achievement goal framework. Journal of Personality and Social Psychology, 80, 501-519.

Elliot, A. J., Murayama, K., \& Pekrun, R. (2011). A $3 \times 2$ achievement goal model. Journal of Educational Psychology, 103, 632-648.

Flett, G. L., Blankstein, K. R., \& Hewitt, P. L. (2009). Perfectionism, performance, and state positive affect and negative affect after a classroom test. Canadian Journal of School Psychology, 24, 4-18.

Flett, G. L., Hewitt, P. L., Oliver, J. M., \& Macdonald, S. (2002). Perfectionism in children and their parents: A developmental analysis. In G. L. Flett \& P. L. Hewitt (Eds.), Perfectionism (pp. 89-132). Washington, DC: APA.

Frost, R. O., Marten, P., Lahart, C., \& Rosenblate, R. (1990). The dimensions of perfectionism. Cognitive Therapy and Research, 14, 449-468.

Hewitt, P. L., \& Flett, G. L. (1991). Perfectionism in the self and social contexts: Conceptualization, assessment, and association with psychopathology. Journal of Personality and Social Psychology, 60, 456-470.

Hewitt, P. L., \& Flett, G. L. (2004). Multidimensional Perfectionism Scale (MPS): Technical manual. Toronto: Multi-Health Systems.

Hill, R. W., Huelsman, T. J., \& Araujo, G. (2010). Perfectionistic concerns suppress associations between perfectionistic strivings and positive life outcomes. Personality and Individual Differences, 48, 584-589.

Kutner, M. H., Nachtsheim, C. J., \& Neter, J. (2004). Applied linear regression models (4th ed.). New York: McGraw-Hill. 
Maltby, J., Day, L., \& Macaskill, A. (2010). Personality, individual differences and intelligence (2nd ed.). Harlow: Pearson.

Moller, A. C., \& Elliot, A. J. (2006). The $2 \times 2$ achievement goal framework: An overview of empirical research. In A. V. Mitel (Ed.), Focus on educational psychology research (pp. 307-326). New York: Nova.

Noftle, E. E., \& Robins, R. W. (2007). Personality predictors of academic outcomes: Big Five correlates of GPA and SAT scores. Journal of Personality and Social Psychology, 93, 116130.

Preacher, K. J., \& Hayes, A. F. (2004). SPSS and SAS procedures for estimating indirect effects in simple mediation models. Behavior Research Methods, Instruments, \& Computers, 36, 717-731.

Siu-Man, N. D., \& Leung, M.-T. (2014). A path analytic model of Chinese-style achievement motivation, $3 \times 2$ achievement goals and self-regulated learning of Hong Kong undergraduates. In Asia Pacific International Academy (Ed.), 2014 Asian Congress of Applied Psychology: Conference proceedings (pp. 292-319). Singapore: Editor.

Slaney, R. B., Rice, K. G., Mobley, M., Trippi, J., \& Ashby, J. S. (2001). The revised Almost Perfect Scale. Measurement and Evaluation in Counseling and Development, 34, 130-145.

Stoeber, J. (2012). Perfectionism and performance. In S. M. Murphy (Ed.), The Oxford handbook of sport and performance psychology (pp. 294-306). New York: Oxford University Press.

Stoeber, J., \& Childs, J. H. (2010). The assessment of self-oriented and socially prescribed perfectionism: Subscales make a difference. Journal of Personality Assessment, 92, 577585.

Stoeber, J., \& Otto, K. (2006). Positive conceptions of perfectionism: Approaches, evidence, challenges. Personality and Social Psychology Review, 10, 295-319.

Stoeber, J., Stoll, O., Salmi, O., \& Tiikkaja, J. (2009). Perfectionism and achievement goals in young Finnish ice-hockey players aspiring to make the Under-16 national team. Journal of Sports Sciences, 27, 85-94.

Stoeber, J., Uphill, M. A., \& Hotham, S. (2009). Predicting race performance in triathlon: The role of perfectionism, achievement goals, and personal goal setting. Journal of Sport \& Exercise Psychology, 31, 211-245.

Tabachnick, B. G., \& Fidell, L. S. (2007). Using multivariate statistics (5th ed.). Boston, MA: Pearson.

Yang, Y., \& Cao, L. (2013). Differential influences of achievement approach goals and intrinsic/extrinsic motivation on help-seeking in e-learning. Knowledge Management \& ELearning, 5, 153-169.

\section{Footnotes}

${ }^{1}$ The third dimension, other-oriented perfectionism, captures perfectionistic expectations directed at others and was therefore disregarded in the present study.

${ }^{2}$ Test available from the corresponding author.

${ }^{3}$ Analyses available from the corresponding author. 
Table 1

Bivariate Correlations, Descriptive Statistics, and Cronbach's Alphas

\begin{tabular}{|c|c|c|c|c|c|c|c|c|c|}
\hline Variable & 1 & 2 & 3 & 4 & 5 & 6 & 7 & 8 & 9 \\
\hline \multicolumn{10}{|l|}{ Perfectionism (T1) } \\
\hline \multicolumn{10}{|l|}{ 1. Self-oriented perfectionism } \\
\hline 2. Socially prescribed perfectionism & $.45^{* * *}$ & & & & & & & & \\
\hline \multicolumn{10}{|l|}{ Achievement goals (T2.1) } \\
\hline 3. Task-approach goals & $.32 * *$ & .07 & & & & & & & \\
\hline 4. Task-avoidance goals & $.29 * *$ & .13 & $.71 * * *$ & & & & & & \\
\hline 5. Self-approach goals & $.31 * *$ & .15 & $.65^{* * *}$ & $.70 * * *$ & & & & & \\
\hline 6. Self-avoidance goals & $.26^{* *}$ & .18 & $.59 * * *$ & $.64 * * *$ & $.79 * * *$ & & & & \\
\hline 7. Other-approach goals & $.49 * * *$ & $.30^{* *}$ & $.64 * * *$ & $.59 * * *$ & $.66^{* * *}$ & $.51^{* *}$ & & & \\
\hline 8. Other-avoidance goals & $.42 * * *$ & .16 & $.61 * * *$ & $.66^{* * *}$ & $.58 * * *$ & $.57^{* *}$ & $.76^{* * *}$ & & \\
\hline 9. Exam performance (T2.2) & $.22^{*}$ & -.12 & $.39 * * *$ & $.21^{*}$ & .14 & $.20^{*}$ & $.28^{* *}$ & $.34 * * *$ & \\
\hline$M$ & 4.82 & 3.76 & 5.08 & 5.40 & 4.38 & 4.87 & 4.22 & 5.07 & 12.96 \\
\hline$S D$ & 0.98 & 0.94 & 1.35 & 1.34 & 1.50 & 1.48 & 1.53 & 1.49 & 3.62 \\
\hline Cronbach's alpha & .92 & .90 & .95 & .91 & .93 & .96 & .96 & .97 & .73 \\
\hline
\end{tabular}

Note. $N=100$. All scores are average scores except exam performance; see $2.4 . \mathrm{T} 1=$ Time 1 ; T2.1 = Time 2, Part 1 ; T2.2 $=$ Time 2, Part 2. Perfectionism (T1) was measured 2-3 days before achievement goals were measured (T2.1) which were measured directly before the exam (T2.2); see 2.2 for details. ${ }^{*} p<.05 .{ }^{* *} p<.01 .{ }^{* * *} p<.001$. 
Table 2

Summary of Regression Analyses Predicting Exam Performance

Criterion $=$ exam performance $(\mathrm{T} 2.2)$

Predictor

$\Delta R^{2}$

$\beta$

Regression Analysis 1

Achievement goals (T2.2)

$.216^{* * *}$

Task-approach goals

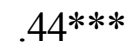

Task-avoidance goals

$-.15$

Self-approach goals

$-.30$

Self-avoidance goals

.12

Other-approach goals

.05

Other-avoidance goals

.25

Regression Analysis 2

Step 1: Perfectionism (T1)

$.106^{* *}$

Self-oriented perfectionism

Socially prescribed perfectionism $.34 * *$

Step 2: Achievement goals (T2.1)

Self-oriented perfectionism

.18

Socially prescribed perfectionism

$-.25 *$

Task-approach goals

Task-avoidance goals

$-.12$

Self-approach goals

$-.35$

Self-avoidance goals

Other-approach goals

Other-avoidance goals

.18

Note. $N=100 . \mathrm{T} 1=$ Time $1 ; \mathrm{T} 2.1=$ Time 2 , Part 1 ; T2.2 $=$ Time 2, Part 2. Perfectionism (T1) was measured 2-3 days before achievement goals were measured (T2.1) which were measured directly before the exam (T2.2); see 2.2 for details.

$* p<.05 . * * p<.01 . * * * p<.001$. 


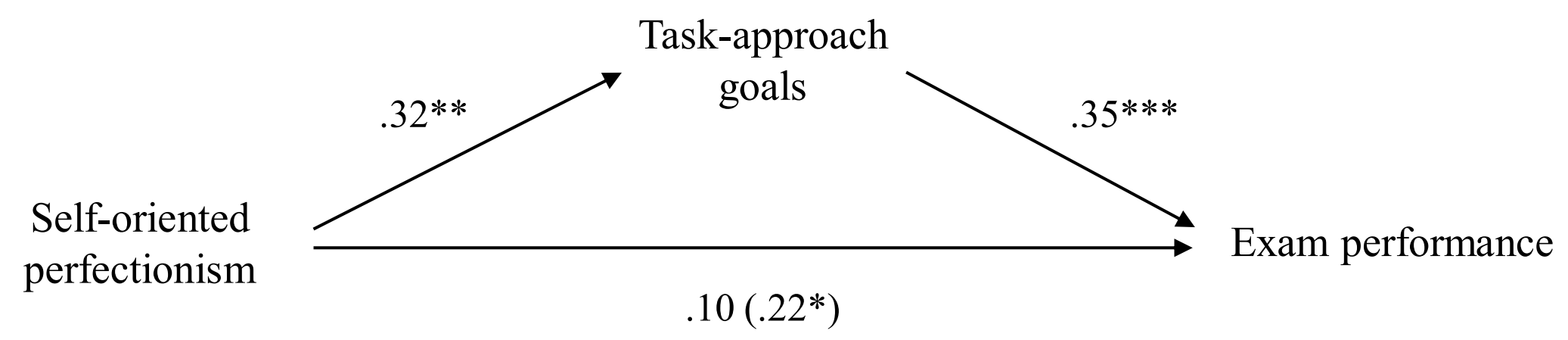

Figure 1. Task-approach goals (T2.1) mediating the relationship between self-oriented perfectionism (T1) and exam performance (T2.2; cf. Table 2). 